\title{
Correlation of Dosimetric Parameters With Subclinical Liver Function Test Alterations After Radiotherapy for Upper Gastrointestinal Tract Tumors
}

\author{
K. Malek ${ }^{1}$, H. Zhang ${ }^{1}$, Y. Boudam ${ }^{1}$, M. Colonna ${ }^{2}$, R. Sihanath ${ }^{1}$, C. Rebischung ${ }^{1} \&$ J. Balosso ${ }^{1,3}$ \\ ${ }^{1}$ Pôle de Cancérologie-Hématologie, Hôpital A.Michallon, CHU de Grenoble, BP 217, GRENOBLE cedex9, \\ France \\ ${ }^{2}$ Registre du Cancer de l'Isère, Meylan, France \\ ${ }^{3}$ Université Joseph Fourier, BP 53, GRENOBLE cedex 9, France \\ Correspondence: K. Malek, Pôle de Cancérologie-Hématologie, Hôpital A.Michallon, CHU de Grenoble, BP \\ 217, F-38043 GRENOBLE cedex9, France. Tel: 33-4-7676-5435. Fax: 33-4-7676-5629. E-mail: \\ JBalosso@chu-grenoble.fr
}

Received: September 4, 2014 Accepted: October 8, 2014 Online Published: October 31, 2014

doi:10.5539/cco.v3n2p70 URL: http://dx.doi.org/10.5539/cco.v3n2p70

\begin{abstract}
Purpose: To characterize conditions of radiation induced liver diseases (RILD) after radiation therapy administered for upper gastrointestinal tract tumours, not involving the liver.

Method \& Materials: this is a retrospective study of 169 patients treated by three dimensional conformal radiations for upper gastrointestinal tract tumours between 2002 and 2008 at the Michallon hospital. Cumulative and differential DVHs (Dose Volume Histogram) were extracted from TPS generated files and liver function tests (ALAT, ASAT, GGT and AP) and tumour markers (CA 19.9 and CEA) from patient records at four different time points (before treatment, and one, three, and six months after the end of treatment). Liver function alteration was defined as an increase in one parameter over 1.5 times the upper normal limit. All these data have been stored and statistically worked out using Lyman model parameters.
\end{abstract}

Results: 34 patient records were studied. Twelve patients had RILD but none at six months. Results are consistent with the Burman description. Dosimetric profiles are different and a correlation appears between RILD and the level of doses given to liver. V40Gy is the most predictive dosimetric parameter for subclinical liver function changes.

Conclusion: While dosimetric parameters are predictive of liver biological disorder, the Lyman model is not predictive of these mild disorders.

Keywords: liver biologic alteration, Radiation-Induced Liver Disease (RilD), NTCP, normal liver irradiated tolerance, predictive factors.

\section{Introduction}

The liver is a rather radiosensitive organ which could be either the target of radiotherapy or, most of the time, an organ at risk for a radiotherapy devoted to a nearby tumor. This is the case for most of the abdominal or lower thoracic irradiations. After exclusive or adjuvant radiotherapy for cancers of the upper gastrointestinal tract such as gastric, bile-duct and pancreatic adenocarcinomas, the interpretation of changes in liver function tests (LFT) during follow-up can be problematic. Although in some patients such changes are a sign of liver metastases, others present persistent and durable LFT abnormalities in the absence of any sign of liver dissemination. Some observers have associated such alterations with visible vascular or density temporary modifications in irradiated liver regions (Sheng et al., 2010), suggesting a possible alternative origin for LFT abnormalities in patients with no sign of metastasis. While radiation-induced liver disease (RILD) is a well-known complication of radiotherapy (Dawson et al., 2002; Lawrence, Robertson et al. 1995; Lawrence, Ten Haken et al. 1992), adherence to published and widely accepted dosimetric guidelines can in general prevent its occurrence (Kutcher, Burman et al., 1991), (Lawrence, Robertson et al., 1995). Elaborating a dose-effect relationship between dose distributions to normal liver and less serious subclinical LFT alterations could help clinicians in interpreting such 
alterations detected during the follow-up of patients having undergone upper abdominal radiation therapy.

We attempt herein to establish such a dose-effect relationship and associated radiobiological parameters for normal liver in a series of patients treated for upper gastrointestinal tract cancers, with no disease involvement of the liver. We assumed, as working hypothesis, that the liver dose volume histogram (DVH) should be predictive of RILD.

\section{Materials and Methods}

\subsection{Type of Study}

This is a retrospective study of patients receiving radiotherapy for upper GI tract tumors between 2002 and 2008 at Grenoble University Hospital, France. Some of these patients were included in previous prospective studies designed for other purposes (GERCOR D98-1 and GERCOR D03-1). Data were retrospectively extracted from the medical and the technical records of all patients.

\subsection{Patients' Inclusion and Exclusion Criteria}

Eligible patients were any receiving radical or adjuvant radiotherapy for upper GI tract cancers, with no history of liver metastasis or other liver disease (e.g. hepatitis, cirrhosis or any liver tumor). Patients for whom no liver function tests were on file for the first year of post-irradiation follow-up were excluded, as were patients with radiological evidence of liver progression, and patients with increasing pre- or post-irradiation tumor markers (CA 19-9 or CEA), for whom possible liver cancer dissemination was considered to be a confounding factor in the liver function test analysis.

\subsection{Principle of Treatment}

All patients received either radical chemo-radiotherapy for inoperable locally advanced non metastatic adenocarcinoma of pancreas (target dose $55 \mathrm{~Gy}$ ), or adjuvant chemo-radiotherapy for completely resected pancreatic carcinomas (target dose $55 \mathrm{~Gy}$ ) or adjuvant chemo-radiotherapy for gastric adenocarcinoma or extra hepatic cholangiocarcinoma (target dose $54 \mathrm{~Gy}$ ). Irradiations were 3D conformational radiotherapy based on a simulation CT and calculated with the same TPS (DOSIGRAY ${ }^{\circledR} 2002$ version, Dosisoft, F-94230 Cachan, France), and managed by the same radiation oncologist (JB). Treatments were carried out with two linacs, either a Siemens Mevatron ${ }^{\circledR} \mathrm{KD} 2$ or an Elekta SLi ${ }^{\circledR}$ producing X-ray beams of 6 or $18 \mathrm{MV}$. Typically 4 photon beams were used (AP-PA and lateral fields) delivering 45 to $54 \mathrm{~Gy}$, with a $10 \mathrm{~Gy}$ boost above $45 \mathrm{~Gy}$, only in case of pancreatic targets. The boost was delivered by 2 or most often 4 beams. Fractionation was 1.8 or 2.25 Gy per session delivered once daily for respectively 5 or 4 days a week. Typically irradiated volumes were very similar and encompassed: i) for gastric cancer, the retro gastric lymph nodes area from the celiac region down to the upper mesenteric artery, the stomach resection bed, the spleen and the liver hila; ii) for bilio-pancreatic tumors, the retro gastric lymph nodes area from the celiac region down to the upper mesenteric vessels, the tumor or the tumor bed, the whole duodenum and the liver hilum, the boost was limited to the CT visible pancreatic tumor with a $2 \mathrm{~cm}$ margin.

Concomitant chemotherapy was administered to all patients either as continuous $5 \mathrm{FU}$ infusion $\left(250-300 \mathrm{mg} / \mathrm{m}^{2} / \mathrm{d}\right.$ for adjuvant gastric adenocarcinomas, extra hepatic cholangiocarcinoma and adjuvant pancreatic carcinoma GERCOR D98-1) or as a combination of weekly administrations of oxaliplatine $\left(60 \mathrm{mg} / \mathrm{m}^{2}\right)$ with a continuous $5 \mathrm{FU}$ infusion $\left(250 \mathrm{mg} / \mathrm{m}^{2} / \mathrm{d}\right)$ for locally advanced pancreatic adenocarcinomas.

\subsection{Dosimetric Parameters and Calculations Means}

Dosimetric parameters were extracted from the treatment planning system (TPS) software DOSIGRAY ${ }^{\circledR} 2002$ version (Dosisoft, F-94230 Cachan, France). The dose calculation algorithm was the Clarkson-Cunningham one, based on the principle of primary and scattered dose separation. For each patient the volume of whole liver, the cumulative DVH (cDVH), the differential DVH (dDVH), and the maximal dose given to the liver were extracted from the TPS. The differential and cumulative DVH for the liver dose distributions were recalculated to have homogeneous data throughout the different cases and uniform dose interval (cDVH) or dose bin of $5 \mathrm{~Gy}(\mathrm{dDVH})$ along the dose range. This interval has been chosen consistently with the usual accuracy of routine dosimetry and a sustainable amount of data that could be thoroughly communicated as in Table 1.

\subsection{Biological Parameters Definition}

LFT perturbation was determined by the study of five parameters: aspartate aminotransferase (ASAT), alanine aminotransferase (ALAT), gamma-glutamyl transferase (GGT), alkaline phosphatase (AP), and bilirubin. These parameters were included on most routine laboratory tests. The combined elevation of these enzymes indicates the presence of liver disease. While AP is mainly produced in the bones and the liver, it is also produced in many 
other organs, such as the intestines, kidneys, and placenta; GGT is produced predominantly in the liver. Therefore, elevated levels of AP are more specific for liver lesions in the presence of concomitant GGT elevations. Conversely, GGT elevations are specific for liver disease regardless of AP levels.

Data on the clinical evolution and LFT profiles were collected for each patient at different times: before treatment, and at one, three, and six months after the treatment.

We considered as abnormal any parameter above 1.5 times the upper physiological limit (quoted as $\mathrm{N}$ ) of the laboratory having done the test. A full set of LFTs was thus considered normal if each parameter was equal or inferior to its $\mathrm{N}$, and as abnormal if at least one of the parameters was above $(1.5 \times \mathrm{N})$. We also took into account the evolution of LFT profiles during the follow-up period: patients whose tests remained abnormal until 6 months were designated $(\mathrm{P}+)$, and patients whose LFTs normalized during the study period were designated (P-). Thus the biological data throughout the study period have been transformed in a discrete parameter $\mathrm{P}-\mathrm{vs} \mathrm{P}+$ or respectively 0 and 1 in the different charts.

\subsection{Radiobiological Model and Statistics}

For each patient the differential DVH was computed using the initial treatment plan. From these differential DVHs were calculated the effective volume (Veff) related to the maximal dose (Dmax) (Lyman 1985), (Lyman 1992) and partial volumes for a series of lower doses segmented as bin of 5 Gy width. These dosimetric data were then correlated with binary LFT values $\mathrm{P}+$ or P- as defined above.

To test the consistency of our data with previously published liver tolerance guidelines, we used the Lyman model, as published by Burman and Emami (Burman, Kutcher et al. 1991, Emami, Lyman et al., 1991) with the liver parameters suggested by Burman $(\mathrm{n}=0.32 ; \mathrm{m}=0.15 ; \mathrm{TD} 50 / 5=40 \mathrm{~Gy})$, to generate multiple series of curves showing on one hand the hyperbolic relationship between Veff and dose, and on the other hand the sigmoid relationship between NTCP and dose (Brenner, 1993; Kutcher, 1996; Kutcher \& Burman, 1989). We then superposed our own data on these curves.

\section{Results}

\subsection{Description of the Population}

Between 2002 and 2008, 169 patients have been treated by three dimensional conformal radiation therapy for upper gastrointestinal tract tumors in our department, among them, 38 patient records were retained for this study. Twelve patients had blood test alteration compatible with a RILD. Patient age ranged from 26 to 82 years at the time of treatment. In all cases, the applied dose-volume constraint for the liver was V25Gy $<60 \%$, according to our departmental guidelines. Maximal doses delivered to PTVs ranged from 45 to $61.25 \mathrm{~Gy}$. All patients were free of metastasis at the time of treatment.

\subsection{Evolution of the Biological Alteration Along the Study Follow-Up}

Among the studied population, $27 \%$ of the patients had some alteration of their LFTs. These alterations appeared progressively during treatment or within 3 months of the end of the treatment. Thereafter, all patients normalized their tests within 6 months. The Figure 1 shows the time related evolution of cytolysis (ASAT and ALAT) which is a part of these alterations.

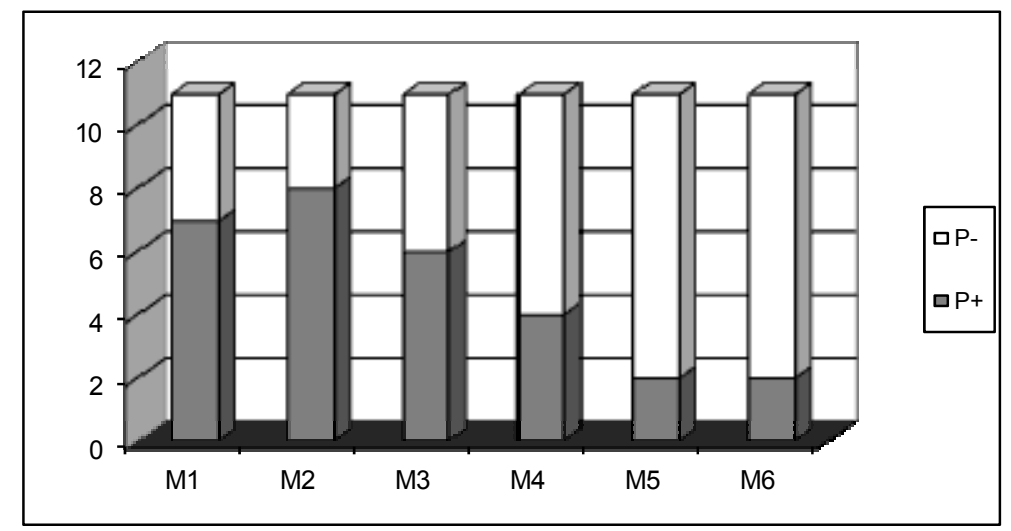

Figure 1. Represents as an example the time related evolution of cytolysis (ASAT and ALAT) quoted as P+ when cytolysis and P- when none at the different time points in months (M1 to M6) versus the number of cases 


\subsection{Biological Data and Effective Volumes for the Maximum Dose}

In Table 1 are listed all of the dose / volume data for the studied population, as calculated and extracted from the cDVH's and the dDVH's. Given the homogeneity of treatment techniques and target doses within the patient group, Veff data were all based on similar Dmax values of $54 \mathrm{~Gy}$, and are therefore possibly strongly correlated to the toxicity of the liver irradiation. The variation in Veff is in fact very low (range $11.13 \mathrm{~mL}$ to $42.02 \mathrm{~mL}$; mean $=26.89, \mathrm{SD}=6.66$ ), and the relation between Veff and LFT alterations is clear.

Table 1. Complete set of the dose/volume data for the studied population as calculated and extracted from the cumulative and the differential DVHs. LFT are quoted as 0 if no biological change and 1 if biological changes, $\mathrm{Vxx}_{\mathrm{Gy}}$ are the successive volumes of liver $(\mathrm{mL})$ receiving more than $\mathrm{xxGy}$, LVol is the liver volume in $\mathrm{mL}$, Dmax in Gy and Veff in \%

\begin{tabular}{|c|c|c|c|c|c|c|c|c|c|c|c|}
\hline LFT & $\begin{array}{l}\mathrm{V} 20_{\mathrm{Gy}} \\
(\mathrm{mL})\end{array}$ & $\mathrm{V} 25_{\mathrm{Gy}}$ & $\mathrm{V} 30_{\mathrm{Gy}}$ & $\mathrm{V} 35_{\mathrm{Gy}}$ & $\mathrm{V} 40_{\mathrm{Gy}}$ & V45 Gy & $\mathrm{V} 50_{\mathrm{Gy}}$ & $\mathrm{V} 55_{\mathrm{Gy}}$ & LVol (mL) & $\begin{array}{l}\text { Dmax } \\
\text { (Gy) }\end{array}$ & $\begin{array}{l}\text { Veff } \\
(\%)\end{array}$ \\
\hline 0 & 426.49 & 377.75 & 316.82 & 194.97 & 146.23 & 121.85 & 97.48 & 24.37 & 1218.5 & 55 & 24.51 \\
\hline 0 & 212.01 & 87.30 & 62.36 & 49.89 & 37.41 & 24.94 & 12.47 & 0 & 1247.1 & 55 & 15.45 \\
\hline 0 & 1018.09 & 962.30 & 669.43 & 557.86 & 516.02 & 488.12 & 446.28 & 111.57 & 1394.6 & 55 & 42.02 \\
\hline 1 & 1073.01 & 940.72 & 440.96 & 382.17 & 338.07 & 235.18 & 117.59 & 102.89 & 1469.8 & 55 & 29.36 \\
\hline 0 & 300.47 & 240.38 & 168.26 & 108.17 & 72.11 & 60.09 & 48.07 & 12.02 & 1201.8 & 56 & 19.23 \\
\hline 0 & 439.51 & 402.89 & 366.26 & 329.63 & 207.55 & 183.13 & 158.71 & 48.83 & 1220.8 & 55 & 27.01 \\
\hline 0 & 706.44 & 694.46 & 431.05 & 251.44 & 239.47 & 215.52 & 203.54 & 83.81 & 1197.3 & 55 & 29.44 \\
\hline 0 & 1168.02 & 1150.05 & 1132.08 & 1096.14 & 1078.17 & 1042.23 & 1006.2 & 826.60 & 1796.9 & 56 & 18.75 \\
\hline 1 & 867.93 & 810.07 & 659.63 & 428.18 & 358.74 & 266.17 & 243.02 & 92.58 & 1157.2 & 57 & 37.99 \\
\hline 1 & 1338.11 & 1240.79 & 851.53 & 656.89 & 608.23 & 583.90 & 510.91 & 48.66 & 2432.9 & 57 & 36.58 \\
\hline 1 & 1631.25 & 1540.62 & 1178.12 & 906.25 & 815.62 & 725.00 & 498.43 & 271.87 & 2265.6 & 56 & 35.82 \\
\hline 1 & 467.70 & 442.42 & 290.73 & 164.33 & 139.05 & 113.76 & 88.48 & 37.92 & 1264.0 & 54 & 22.81 \\
\hline 0 & 701.28 & 539.45 & 364.13 & 229.27 & 188.81 & 148.35 & 80.91 & 13.49 & 1348.6 & 57 & 27.81 \\
\hline 0 & 1118.42 & 1070.49 & 399.43 & 319.55 & 239.66 & 191.73 & 159.7 & 31.95 & 1597.7 & 57 & 31.68 \\
\hline 0 & 576.12 & 535.92 & 495.73 & 482.33 & 415.34 & 254.56 & 227.76 & 13.40 & 1339.8 & 57 & 25.42 \\
\hline 0 & 560.80 & 498.49 & 327.13 & 249.24 & 233.67 & 186.93 & 46.73 & 15.58 & 1557.78 & 58 & 24.97 \\
\hline 0 & 749.01 & 726.65 & 458.35 & 413.63 & 391.27 & 357.73 & 301.84 & 223.58 & 1117.9 & 56 & 26.42 \\
\hline 0 & 753.51 & 384.77 & 368.74 & 352.71 & 320.64 & 176.35 & 160.32 & 48.10 & 1603.2 & 56 & 25.84 \\
\hline 1 & 787.98 & 722.31 & 630.38 & 433.39 & 393.99 & 354.59 & 315.19 & 105.06 & 1313.3 & 55 & 35.20 \\
\hline 1 & 490.29 & 408.57 & 277.83 & 245.14 & 212.46 & 114.40 & 32.69 & 0 & 1634.2 & 56 & 23.08 \\
\hline 1 & 621.42 & 514.28 & 171.43 & 150.00 & 117.86 & 85.71 & 53.57 & 21.43 & 1071.4 & 56 & 25.29 \\
\hline 0 & 745.63 & 697.53 & 324.71 & 276.60 & 252.55 & 216.47 & 113.05 & 57.73 & 1202.6 & 56 & 26.38 \\
\hline 1 & 669.11 & 610.42 & 563.46 & 539.98 & 493.03 & 422.60 & 363.90 & 140.86 & 1173.8 & 57 & 36.89 \\
\hline 1 & 1010.98 & 520.36 & 446.02 & 401.42 & 327.08 & 267.61 & 223.01 & 178.40 & 1486.7 & 57 & 22.86 \\
\hline 0 & 701.47 & 643.86 & 331.29 & 250.63 & 213.18 & 161.32 & 72.02 & 27.37 & 1440.4 & 57 & 20.56 \\
\hline 0 & 504.11 & 562.73 & 502.94 & 378.67 & 310.68 & 248.54 & 157.10 & 84.41 & 1172.3 & 58 & 28.56 \\
\hline 1 & 663.02 & 421.92 & 337.54 & 301.37 & 277.26 & 216.99 & 168.76 & 96.44 & 1205.4 & 57 & 25.61 \\
\hline 1 & 407.17 & 308.13 & 154.07 & 132.06 & 121.05 & 88.04 & 66.03 & 22.01 & 1100.4 & 57 & 21.75 \\
\hline 0 & 802.31 & 744.79 & 499.55 & 313.36 & 274.00 & 207.394 & 68.12 & 18.17 & 1513.8 & 56 & 27.62 \\
\hline 0 & 1297.84 & 1261.48 & 785.25 & 523.50 & 478.06 & 438.07 & 341.73 & 229.03 & 1817.7 & 59 & 11.13 \\
\hline 0 & 1045.32 & 975.63 & 783.99 & 484.33 & 442.52 & 350.18 & 325.79 & 130.66 & 1742.2 & 57 & 27.53 \\
\hline 0 & 840.77 & 562.32 & 427.14 & 389.30 & 364.962 & 324.41 & 183.83 & 8.11 & 1351.7 & 56 & 31.91 \\
\hline 0 & 1096.24 & 687.78 & 381.43 & 355.90 & 249.282 & 217.75 & 192.22 & 138.15 & 1501.7 & 56 & 26.02 \\
\hline 0 & 728.06 & 575.64 & 173.14 & 143.54 & 122.822 & 76.95 & 29.60 & 0 & 1479.7 & 46 & 21.39 \\
\hline
\end{tabular}




\subsection{Correlation Between the Effective Volumes or Dose-Volumes Parameters and the Risk of Biological Alterations}

The Figure 2 shows the variation of the multiple regression coefficients of the eight Vdose independent values versus the risk of biological alteration. The V40Gy was the most predictive volume for LFT elevation in this specific population treated with the described protocols. This characteristic probably cannot be generalized.

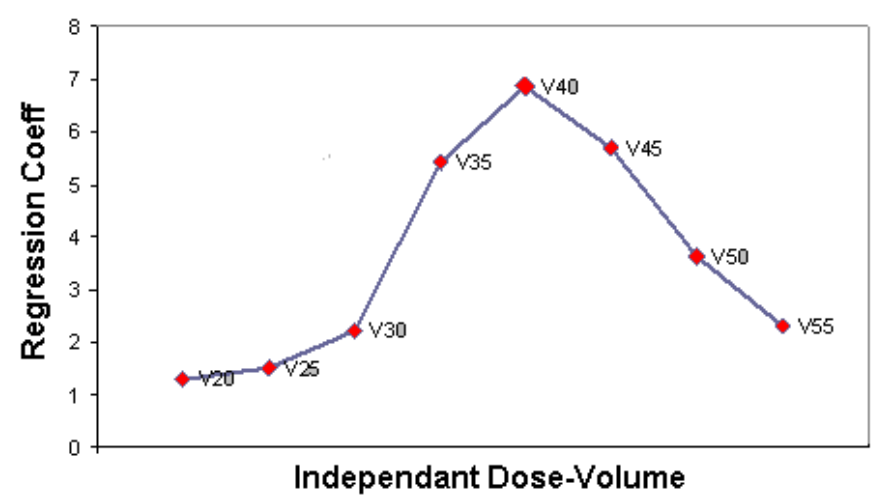

Figure 2. Display of the variation of the multiple regression coefficients of the eight Vdose independent values versus the discrete risk of biological alteration. The V40Gy is the most predictive volume for LFT elevation in this specific population

The distribution of LFT elevation according to V40Gy or Veff (calculated for a Dmax of 54Gy) is shown in Figure 3. While no elevations appeared for V40Gy values lower than $10 \%$ (area A), any irradiation producing a V40Gy higher than $38 \%$ is expected to produce alterations (area C). Between these upper and lower limits (area B), the interpretation of the origin of LFT alterations is difficult, since a clear correlation with radiation dose is unclear. Similarly, for Veff lower than $21 \%$ (area A) we found no LFT elevations, whereas with a Veff above $32.5 \%$ (area C) changes were almost certain, with an indefinite area (B) between 21 and 32.5\%.
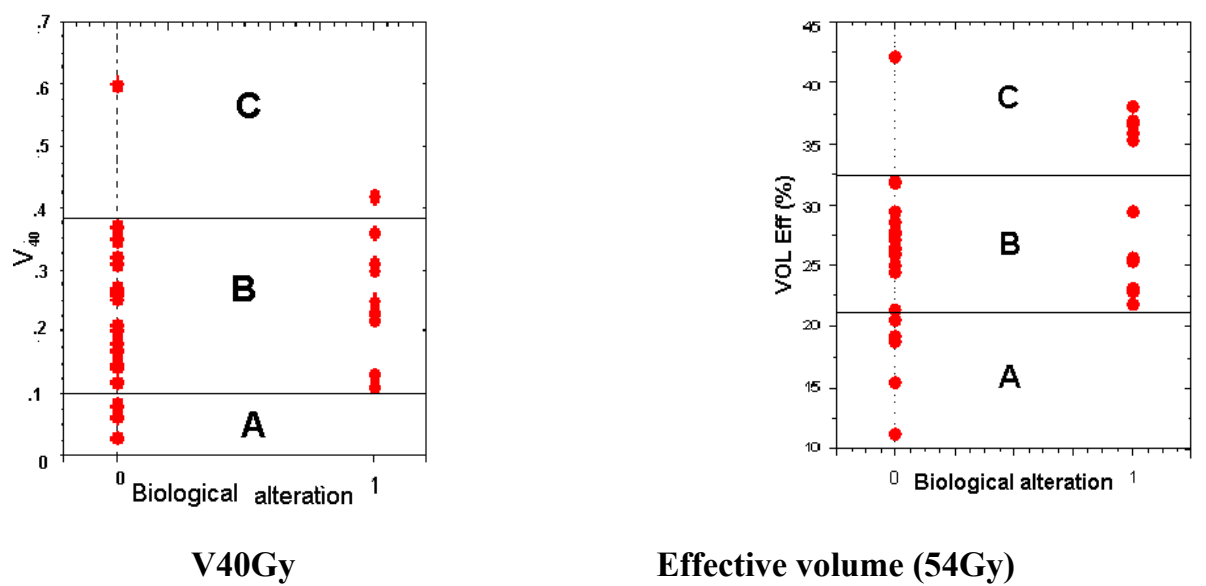

\section{Effective volume (54Gy)}

Figure 3. Distributions of LFT elevation according to V40Gy or Veff (for Dmax $=54 \mathrm{~Gy}$ ). While no elevations appeared for V40Gy values lower than $10 \%$ (area A), any irradiation producing a V40Gy higher than $38 \%$ is expected to produce alterations (area $\mathrm{C}$ ). Between these upper and lower limits (area B), the interpretation of the origin of LFT alterations is ambiguous. Similarly, for Veff lower than 21\% (area A) there are no LFT elevations, whereas with a Veff above $32.5 \%$ (area C) changes were almost certain, with an indefinite area (B) between 21 and $32.5 \%$

Given the above, we propose that LFT changes appearing in area A (V40Gy $<10 \%$ or Veff $54 \mathrm{~Gy}, 21 \%$ ) should be attributed to some liver disease or disorder independent of the irradiation and deserve specific hepato-biliary investigations; conversely, biological disorders appearing in area $\mathrm{C}$ after an irradiation producing a V40Gy > $38 \%$ or a $\operatorname{Veff}_{54 \mathrm{~Gy}}>32.5 \%$ can almost certainly be, at least partially, attributed to the irradiation and may at first 
be simply followed, with a spontaneous decrease expected in case of RILD.

\subsection{Row Comparison of the Risk of Biological Alteration and the Lyman's NTCP Prediction}

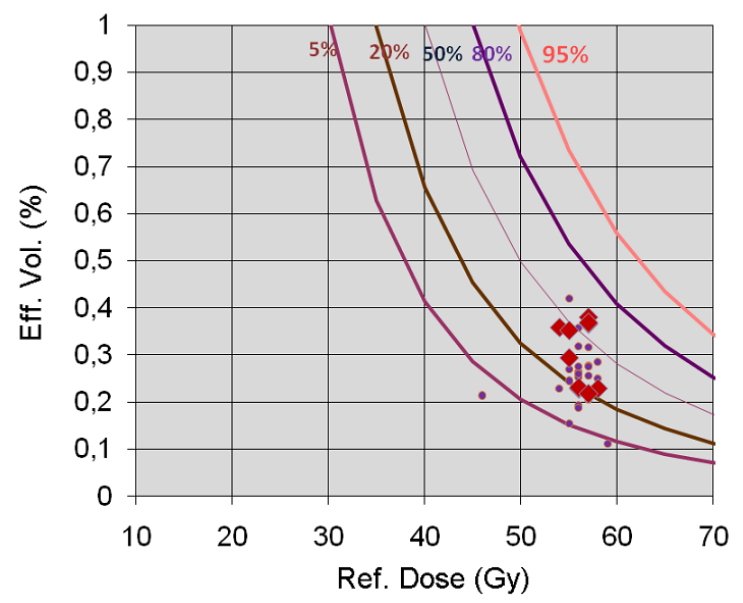

Figure 4. Iso-NTCP chart of the liver according to Emami, Burman and Kutcher, correlating the Eff.Vol to the reference dose with the superposition of the data of the present study which are clustered in the area between $20 \%$ and $50 \%$ risk at 5 years. On this chart the cases with LFT toxicity are represented as red diamonds

As it can be seen on Figure 4, our data set can be represented on an iso-NTCP chart of the liver and are clustered in the area between $20 \%$ and $50 \%$ risk at 5 years. On this chart the cases with LFT toxicity represented as red diamonds are not obviously differently located than the other cases. Although this chart is parameterized to represent neither the low biological risk (and not serious late effects) nor the earlier timeframe of our study (effects observed in the 6 first month of follow-up), the predicted high risk for most of our patients for late RILD is worth noting, but does not correlate at all with our clinical experience which is a complete lack of symptomatic late effects in this population (data not shown).

\section{Discussion}

While previous works on the subject of liver toxicity of radiation have largely used thresholds for serious RILD seen in series of patients treated with older radiation techniques in order to establish the tolerance model for this organ (Austin-Seymour et al., 1986; Lawrence, Robertson et al. 1995), our work is based on current 3-D conformal practice and thus with potentially very different criteria for acceptable liver toxicity in upper abdominal radiotherapy treatments. However, recent comparison between dose-volume results for upper abdominal malignancies between 3DCRT and IMRT have not shown any favourable differences concerning healthy liver irradiation (Nour et al., 2013). Thus we can expect that our data could have some interest even for more advanced irradiation technics than those used in this study.

It is foreseeable that increasingly numerous liver pathologies will be treated by some radiation therapy approaches, necessitating a finer biological semiology, and raising new questions about liver tolerance, including potential long-term consequences of subclinical liver-function changes, the expected time course and evolution of such alterations, and situations in which we can safely observe them without fearing liver cancer relapse.

In this work we have tried to answer some of these questions. Although the small number of cases $(n=34)$ and the homogeneity of the treatment protocols made it impossible to elaborate a model covering a large range of dose-volume conditions, we can nonetheless draw from this work the information that the V40Gy is the most predictive parameter for biological reactions of the liver in our patient population, and that the effective volume, which is a straightforward parameter obtained from any modern TPS, is also quite predictive of the biological risk. An Eff.Vol for $54 \mathrm{~Gy}$ above $21 \%$ is compatible with radiation-induced biological alteration of the liver and an Eff.Vol for 54 Gy above $32 \%$ is almost always associated with biological changes. The Eff.Vol correlation is possibly more independent from our population than the V40Gy correlation which is a kind of characteristic of our treatment protocols and related dose distributions into the liver. A similar study has been carried out in Japan (Tanaka et al., 2013) for primary gastric lymphoma treated by chemotherapy followed by radiotherapy. The level of LFT change for RILD definition was higher than in our study $(2 \times \mathrm{N}$ versus $1.5 \times \mathrm{N})$ and the probability of 
RILD has been studied regarding the VxxGy above which the RILD appeared. The sensitivity of liver appeared higher in this study than in ours, for instance a V20Gy $>30 \%$ was a condition of RILD. The role of chemotherapy could be discussed. In our study the chemotherapy had a lower toxicity level (5FU \pm oxaliplatine vs rituximab + cyclophosphamide + doxorubicin + vincristine) and, regarding the literature and our results, the chemotherapy administered to our patients seamed not alter the liver radio-sensitivity.

The fact that these results have been obtained with a population having no liver disease at all makes a deep difference with the results obtained by other authors with patients treated for liver disease, namely unresectable hepatocellular carcinoma. Actually radiobiological data obtained for such patients are showing a very higher sensitivity of the remaining liver that preclude any possibility to pool such data with ours. Son et al. found, based on the increased cirrhosis score, that the toxicity after helical irradiation appeared with a cutoff value of $43.2 \%$ for the V15Gy 25Gy (Son, Kay et al., 2013) which is substantially lower than the recommendation of keeping the $\mathrm{V} 25 \mathrm{~Gy}<60 \%$, in other words having at least $40 \%$ of the liver below $25 \mathrm{~Gy}$. Consequently, the radiobiologic parameters as $\alpha / \beta=8$ (Son, Jang et al., 2013) or $\mathrm{n}(1), \mathrm{m}(0.36$ or 0.41$)$ and $\mathrm{f}(0.156$ or 0.0$)$ as long as $\mathrm{TD}_{50 / 5}(40.3$ or 24Gy) with $\alpha / \beta=2$ for the Lyman model (Tai et al., 2009) obtained with hepatocellular carcinoma cases with altered hepatic functions are not applicable to healthy liver.

\section{Conclusion}

The present results can help in the interpretation of biological liver disturbances detected during the follow-up of cancer patients with initially healthy liver but at risk of other liver disorders. Our data also shows that the time course of benign post-irradiation biological alterations should be limited to 6 months, which is consistent with the course of imaging alterations of the liver observed after irradiation (Sheng et al., 2010).

Beyond these practical considerations, our study raises the question of the relevance of these biological alterations to long-term risk of more severe liver dysfunction, since the data are "located" in a high-risk NTCP area for later organic RILD (Figure 4). Accordingly, it could be suggested that the observation of biological alterations could be an early indicator of more serious organic risk at a later time, beyond 5 years of follow-up, which is however rarely observed in such diseases, and certainly not at the level of probability foreseen by Emami model.

To go forward in this domain, a new model should be established, adapted to biological assessment (Buffa et al., 2001) incorporating more numerous data from different irradiation protocols with varying target doses and taking carefully account of the functional status of the liver and the administration of chemotherapy. In order to contribute to such future studies, we include in full all of our data in Table 1.

\section{Disclosure}

All authors confirm their co-authorship and declare that have no conflict of interest with any part of contain of this paper and approve the submission.

\section{References}

Austin-Seymour, M. M., Chen, G. T., Castro, J. R., Saunders, W. M., Pitluck, S., Woodruff, K. H., \& Kessler, M. (1986). Dose volume histogram analysis of liver radiation tolerance. Int J Radiat Oncol Biol Phys, 12(1), 31-35. http://dx.doi.org/10.1016/0360-3016(86)90412-8

Brenner, D. J. (1993). Dose, volume, and tumor-control predictions in radiotherapy. Int J Radiat Oncol Biol Phys, 26(1), 171-179. http://dx.doi.org/10.1016/0360-3016(93)90189-3

Buffa, F. M., Davidson, S. E., Hunter, R. D., Nahum, A. E., \& West, C. M. (2001). Incorporating biologic measurements $(\mathrm{SF}(2), \mathrm{CFE})$ into a tumor control probability model increases their prognostic significance: a study in cervical carcinoma treated with radiation therapy. Int J Radiat Oncol Biol Phys, 50(5), 1113-1122.

Burman, C., Kutcher, G. J., Emami, B., \& Goitein, M. (1991). Fitting of normal tissue tolerance data to an analytic function. Int $J$ Radiat Oncol Biol Phys, 21(1), 123-135. http://dx.doi.org/10.1016/0360-3016(91)90172-Z

Dawson, L. A., Normolle, D., Balter, J. M., McGinn, C. J., Lawrence, T. S., \& Ten Haken, R. K. (2002). Analysis of radiation-induced liver disease using the Lyman NTCP model. Int J Radiat Oncol Biol Phys, 53(4), 810-821. http://dx.doi.org/10.1016/S0360-3016(02)02846-8

Emami, B., Lyman, J., Brown, A., Coia, L., Goitein, M., Munzenrider, J. E., Shank, B., Solin, L. J., \& Wesson, M. (1991). Tolerance of normal tissue to therapeutic irradiation. Int J Radiat Oncol Biol Phys, 21(1), 109-122. http://dx.doi.org/10.1016/0360-3016(91)90171-Y 
Kutcher, G. J. (1996). Quantitative plan evaluation: TCP/NTCP models. Front Radiat Ther Oncol, 29, 67-80.

Kutcher, G. J., \& Burman, C. (1989). Calculation of complication probability factors for non-uniform normal tissue irradiation: the effective volume method. Int J Radiat Oncol Biol Phys, 16(6), 1623-1630. http://dx.doi.org/10.1016/0360-3016(89)90972-3

Kutcher, G. J., Burman, C., Brewster, L., Goitein, M., \& Mohan, R. (1991). Histogram reduction method for calculating complication probabilities for three-dimensional treatment planning evaluations. Int $J$ Radiat Oncol Biol Phys, 21(1), 137-146. http://dx.doi.org/10.1016/0360-3016(91)90173-2

Lawrence, T. S., Robertson, J. M., Anscher, M. S., Jirtle, R. L., Ensminger, W. D., \& Fajardo, L. F. (1995). Hepatic toxicity resulting from cancer treatment. Int J Radiat Oncol Biol Phys, 31(5), 1237-1248. http://dx.doi.org/10.1016/0360-3016(94)00418-K

Lawrence, T. S., Ten Haken, R. K., Kessler, M. L., Robertson, J. M., Lyman, J. T., Lavigne, M. L., ... Lichter, A. S. (1992). The use of 3-D dose volume analysis to predict radiation hepatitis. Int J Radiat Oncol Biol Phys 23(4), 781-788. http://dx.doi.org/10.1016/0360-3016(92)90651-W

Lyman, J. T. (1985). Complication probability as assessed from dose-volume histograms. Radiat Res Suppl, 8 , S13-19.

Lyman, J. T. (1992). Normal tissue complication probabilities: variable dose per fraction. Int J Radiat Oncol Biol Phys 22(2), 247-250. http://dx.doi.org/10.1016/0360-3016(92)90040-O

Nour, A. A., Alaradi, A., Mohamed, A., Altuwaijri, S., \& Rudat, V. (2013). Intensity modulated radiotherapy of upper abdominal malignancies: dosimetric comparison with 3D conformal radiotherapy and acute toxicity. Radiat Oncol, 8(1), 207. http://dx.doi.org/10.1186/1748-717X-8-207

Sheng, Y., Wang, Q., Li, Z., Zheng, N., Pei, Y., Chen, L., \& Hu, D. (2010). Time-dependent changes in CT of radiation-induced liver injury: a preliminary study in gastric cancer patients. J Huazhong Univ Sci Technolog Med Sci, 30(5), 683-686. http://dx.doi.org/10.1007/s11596-010-0565-7

Son, S. H., Jang, H. S., Lee, H., Choi, B. O., Kang, Y. N., Jang, J. W., ... Kay, C. S. (2013). Determination of the alpha/beta ratio for the normal liver on the basis of radiation-induced hepatic toxicities in patients with hepatocellular carcinoma. Radiat Oncol, 8, 61. http://dx.doi.org/10.1186/1748-717X-8-61

Tai, A., Erickson, B., \& Li, X. A. (2009). Extrapolation of normal tissue complication probability for different fractionations in liver irradiation. Int $J$ Radiat Oncol Biol Phys, 74(1), 283-289. http://dx.doi.org/10.1016/j.jijobp.2008.11.029

Tanaka, H., Hayashi, S., Ohtakara, K., and Hoshi, H. (2013). Hepatic dysfunction after radiotherapy for primary gastric lymphoma. J Radiat Res, 54(1), 92-97. http://dx.doi.org/10.1093/jrr/rrs062

\section{Copyrights}

Copyright for this article is retained by the author(s), with first publication rights granted to the journal.

This is an open-access article distributed under the terms and conditions of the Creative Commons Attribution license (http://creativecommons.org/licenses/by/3.0/). 